\title{
Search for a diffuse flux of cosmic neutrinos with the ANTARES telescope
}

\author{
Thomas Eberl* \\ Friedrich-Alexander-Universität Erlangen-Nürnberg, Erlangen Centre for Astroparticle Physics, \\ Erwin-Rommel-Str. 1, 91058 Erlangen, Germany \\ E-mail: Thomas.Eberlefau.de
}

\section{Luigi Antonio Fusco}

INFN - Sezione di Bologna and Dipartimento di Fisica e Astronomia dell'Universitá di Bologna, Viale Berti-Pichat 6/2, 40127 Bologna, Italy

E-mail: luigiantonio.fusco@bo.infn.it

\section{Sergio Navas}

Departamento de Fisica Teorica y del Cosmos, Universidad de Granada, Av. Severo Ochoa, 18071 Granada, Spain.

E-mail: navas@ugr.es

\section{Federico Versari}

INFN - Sezione di Bologna and Dipartimento di Fisica e Astronomia dell'Universitá di Bologna, Viale Berti-Pichat 6/2, 40127 Bologna, Italy

E-mail: federico.versariabo.infn.it

\section{on behalf of the ANTARES Collaboration}

The ANTARES detector is the largest neutrino telescope in the Northern hemisphere. Interactions of all neutrino flavours can be observed and reconstructed by the experiment. Events induced by muon neutrino charged-current interactions represent the track channel: the long path travelled by muons before being detected allows to increase the effective detector volume, with excellent angular resolution but limited precision in the event energy reconstruction. The shower channel, i.e. all neutral-current and charged-current interactions of $v_{e}$ (and possibly $v_{\tau}$ ) allows for a better energy resolution than the track channel, though only events within a limited fiducial volume can be considered. Exploiting its multi-year dataset, the complementarity of its field of view and its excellent reconstruction performance, ANTARES can independently investigate the cosmic neutrino flux reported by the Antarctic IceCube detector. The latest results of these all-flavour searches for a diffuse astrophysical neutrino flux are presented in this contribution. Using events observed in both channels, an excess of events with respect to the expected atmospheric background is observed with a significance below $2 \sigma$. Once systematics are included, the corresponding $68 \%$ confidence interval encloses the observed IceCube signal.

35th International Cosmic Ray Conference - ICRC2017

10-20 July, 2017

Bexco, Busan, Korea

${ }^{*}$ Speaker. 


\section{Introduction}

Astrophysical high-energy neutrinos are probes to investigate the origin, the sources and the acceleration mechanisms of primary cosmic rays (CRs). Neutrinos can indeed be produced at the sources of ultra high-energy cosmic rays (UHECRs) and then travel without deflection or absorption over cosmic distances. CRs can produce neutrinos when interacting with the ambient medium via inelastic collisions on nucleons or photons. The former represents the so-called $p p$ reaction, where a large amount of secondaries is produced, such as short-lived mesons decaying into neutrinos [1]; the latter is described by photo-production processes where the $p \gamma$ reaction produces a $\Delta$-resonance which decays into pions. Neutrinos are produced in the pion decay chain [2]. Under the assumption that neutrinos are produced in meson decays, the neutrino flux at the source is distributed between the three neutrino flavours as $v_{e}: v_{\mu}: v_{\tau}=1: 2: 0$. Vacuum oscillations over cosmic distances would produce equipartition in the three flavours at Earth.

A diffuse flux of cosmic neutrinos might originate from either the ensemble of unresolved individual sources, which are too faint to be detected individually, or from the interaction of highenergy CRs as they propagate in the Universe. The observation of a diffuse flux of cosmic neutrinos, i.e. the measurement of its spectrum and flavour composition, could provide valuable information on their production mechanisms. This can be eventually related to the $\mathrm{CR}$ acceleration and interaction properties. Under the assumptions made by Waxman and Bahcall [3], the diffuse neutrino flux should follow the same spectrum as that of the parent CRs at the acceleration site, i.e. proportional to $E^{-2}$ as expected from the Fermi acceleration mechanism [4]. A softer spectrum might originate from extragalactic accelerators that are inside galaxies which are opaque to CRs. This is the case, for example, for starburst galaxies [5] where the matter density is high enough for protons to lose energy before producing pions. The corresponding cosmic neutrino energy spectrum is steeper and lower in normalisation compared to the Waxman and Bahcall prediction.

Searches for a diffuse flux of cosmic neutrinos from the IceCube collaboration have provided the observation of an excess of neutrinos over the expected background [6, 7]. The measured flux can be modelled with single-power-laws $d N_{v} / d E_{v}=\Phi_{0} E_{v}^{-\Gamma}$, with relatively soft spectral indices $(\Gamma>2)$. Assuming that the astrophysical neutrino flux is isotropic over the whole sky and consisting of equal flavours at Earth, the best-fit spectral index is $\Gamma=2.50 \pm 0.09$ and the normalization at $100 \mathrm{TeV}$ is $\Phi_{0}^{3 f}(100 \mathrm{TeV})=6.7_{-1.2}^{+1.1} \cdot 10^{-18} \mathrm{GeV}^{-1} \mathrm{~cm}^{-2} \mathrm{~s}^{-1} \mathrm{sr}^{-1}$ for an allflavour flux ( $3 f$ ) [7]. Always in IceCube, a separate measurement of muons induced by neutrinos coming from the Northern hemisphere [8] yields a best-fit, single-flavour flux $\Phi_{0}^{1 f}(100 \mathrm{TeV})=$ 9. $0_{-2.7}^{+3.0} \cdot 10^{-19} \mathrm{GeV}^{-1} \mathrm{~cm}^{-2} \mathrm{~s}^{-1} \mathrm{sr}^{-1}$ and $\Gamma=2.13 \pm 0.13$ [8]. This particular channel can access neutrinos only at energies above $100 \mathrm{TeV}$ because of the more abundant atmospheric background from $v_{\mu}$-induced events at lower energies, while analyses including $v_{e}$ and $v_{\tau}$ interactions have lower energy thresholds. These different measurements of the cosmic neutrino flux by IceCube might be a hint to multiple components in the IceCube signal, such as a hard/extragalactic plus a soft/galactic component [9].

The ANTARES detector [10] can provide valuable information for the identification of this signal. This is especially true if part of this signal has a Galactic origin. This possible component is indeed expected to be more intense at lower energies with respect to the predictions for extragalactic fluxes. Because of its lower energy threshold, ANTARES can study in detail this flux either in 
searches for point-like sources [11] or in extended region searches, such as for a contribution of neutrinos coming from CR propagation and interactions in the Milky Way [12].

Previous searches for a diffuse flux of cosmic neutrinos with ANTARES data did not observe any significant excess over the atmospheric background expectations $[13,14,15,16]$. The analysis presented in this contribution extends the previously analysed data sample and uses events from interactions of all neutrino flavours, which improves the sensitivity of the analysis. As a result, an excess of events over the expected atmospheric backgrounds is observed; though this excess is compatible with the background within its uncertainties, it is also compatible with the expectations from a cosmic contribution to the overall event rate.

\section{The ANTARES detector and data sample}

The ANTARES underwater neutrino telescope [10] is located $40 \mathrm{~km}$ off the Southern coast of France in the Mediterranean Sea $\left(42^{\circ} 48^{\prime} \mathrm{N}, 6^{\circ} 10^{\prime} \mathrm{E}\right)$. It consists of a three-dimensional array of 10-inch photomultiplier tubes (PMTs) distributed along 12 vertical lines, anchored to the sea-bed at a depth of about $2500 \mathrm{~m}$ and kept taut by a top buoy. Each line includes 75 PMTs distributed in 25 storeys and facing $45^{\circ}$ downwards. ANTARES is currently the largest and longest operating under-sea neutrino telescope and the largest neutrino experiment in the Northern Hemisphere.

Cherenkov light is induced in the medium by relativistic charged particles produced in the neutrino interaction. Cherenkov photons impinging on the PMTs produce signals ("hits") which are collected by the experiment. The position, time and collected charge of the hits are used to infer the direction and energy of the incident neutrino. The event-wise information is reconstructed starting from the hit information. Events induced by charged-current (CC) interactions of muon neutrinos produce a track signature in the detector; the long extension of the track enlarges the effective volume of the detector, increasing the event rate. For these events a median angular resolution of $0.4^{\circ}$ is achieved [11, 17], though the limited information on the whole event limits the energy resolution [18]. On the other hand, all-flavour neutral-current (NC) and CC $v_{e}$ and $v_{\tau}$ interactions produce electromagnetic and hadronic showers in the instrumented volume. These events can be properly reconstructed with ANTARES [19] only in a restricted fiducial volume, showing a median angular resolution of the order of $3^{\circ}$ and an excellent energy resolution of about $10 \%$.

ANTARES data, collected from 2007 to 2015, are used in this analysis to search for an excess of cosmic neutrinos over the atmospheric backrgrounds. This data sample corresponds to an equivalent livetime of 2450 days, extending the livetime of all previous diffuse-flux analyses performed with ANTARES data by more than a factor of two. Monte Carlo simulations are used to define the event selection. Since data acquisition conditions in the sea environment are not stable in time, dedicated simulations reproduce the time-dependent behaviour of the data taking [20]. Thus, for each data acquisition run, a corresponding Monte Carlo file is generated. A blinding policy is followed for the analysis: $10 \%$ of the collected data is used to check the agreement between data and Monte Carlo in order to avoid biases in the event selection that could otherwise arise from looking directly at data. 


\section{Search method}

The strategy to search for a diffuse flux of cosmic neutrinos can be summarised as a two-step procedure: at first the largely overabundant background of atmospheric muons is reduced by applying cuts on the direction and the quality of the reconstruction output; then, taking advantage of the different spectral behaviour of signal and atmospheric neutrino background, an energy-related cut is applied to improve the signal-to-noise ratio. As mentioned before, equipartition in the three neutrino flavours, as well as between neutrinos and anti-neutrinos, is assumed. In addition, an isotropic flux over the whole sky is considered. Single-power-law energy spectra are used to describe cosmic neutrino fluxes. In this work two options have been considered: $\Gamma=2.0$ and $\Gamma=2.5$, close to the best-fit options for the IceCube cosmic signal.

The most abundant background in the search for neutrinos stems from atmospheric downwardgoing muons reaching the apparatus. The MUPAGE [21, 22] simulation code is used to produce large Monte Carlo samples of atmospheric muon bundles to study this background. The Earth can be used as a shield to reduce the influence of these muons, by rejecting events reconstructed as downward-going. Nonetheless, a certain amount of atmospheric muon events could still be misreconstructed as upward-going. A selection on the event reconstruction quality allows to reduce their influence.

Atmospheric neutrinos are produced together with muons, and they cannot be shielded by the Earth. At high energies, two spectral components contribute to the total atmospheric neutrino flux, namely the conventional and the prompt components. The conventional flux consists of neutrinos produced in the decays of pions and kaons, more abundant but softer in spectrum; the prompt neutrino component is expected to originate from the decays of charmed hadrons in atmospheric particle cascades, thus has a smaller intensity but a harder spectrum. The assumption made here is that the conventional component is described by the Honda et al. calculations [23], while the prompt component follows the Enberg et al. prescription [24]. Different predictions for conventional and prompt fluxes have been taken into account in the evaluation of systematic effects, as described in section 4. ANTARES data have been already used to measure the atmospheric muon neutrino energy spectrum in [25].

Atmospheric neutrino interactions are topologically indistinguishable from cosmic neutrinos in ANTARES. However, at the highest energies the astrophysical neutrino flux should be more intense and possibly become visible. An energy-related selection is defined to maximize the signalto-noise ratio, following the Model Rejection Factor (MRF) procedure [26] based on the Feldmann and Cousins upper limit estimation [27].

\subsection{Neutrinos inducing tracks}

Track-like events are reconstructed through a multi-step procedure based on likelihood fits [17] using PMT hits. The discrimination between downward-going atmospheric muons and high-energy neutrino-induced events is accomplished by selecting events with a low angular error estimate $(\beta)$ and good track-fit quality parameter $(\Lambda)$, as well as a large number of hits correlated with the reconstructed track $\left(N_{\text {hit }}\right)$. An a priori cut on the reconstructed zenith angle $\left(\theta^{\text {track }}>90^{\circ}\right)$ is applied to remove the atmospheric muons which are properly reconstructed as downward-going. Then, a combined cut on $\Lambda, \beta$ and $N_{\text {hit }}$ is used to reject wrongly-reconstructed muons. The optimised cut 
corresponds to $\Lambda>-4.9, \beta<0.5^{\circ}$ and $N_{\text {hit }}>35$; this reduces by 8 orders of magnitude the number of atmospheric muons in the sample with respect to the overall number of reconstructed events. The resulting atmospheric neutrino rate is of about 1 event/day.

The separation between cosmic and atmospheric neutrinos is achieved by estimating the neutrino energy. Even though this is not directly measurable, the neutrino energy is correlated with the number of photons detected in the event. Higher energy neutrinos produce larger energy deposits in the detector, detectable as additional light, which mostly comes from Cherenkov emission by secondary particles produced in catastrophic energy loss processes along the track. An energy estimator based on Artificial Neural Networks [18] provides the best discrimination between atmospheric and cosmic neutrinos. Figure 1 shows the energy estimator distribution for the selected neutrino sample, also showing the optimal selection cut as from the MRF procedure $\left(E_{A N N}>5\right)$ when optimising for a cosmic flux proportional to $E^{-2}$; because of the intense atmospheric muon neutrino background, the optimal selection is almost independent with respect to the spectral behaviour of the cosmic signal. 13.5 \pm 4 background events are expected, while 3-3.5 events should be induced by an IceCube-like signal, depending on the spectral index and flux normalisation.
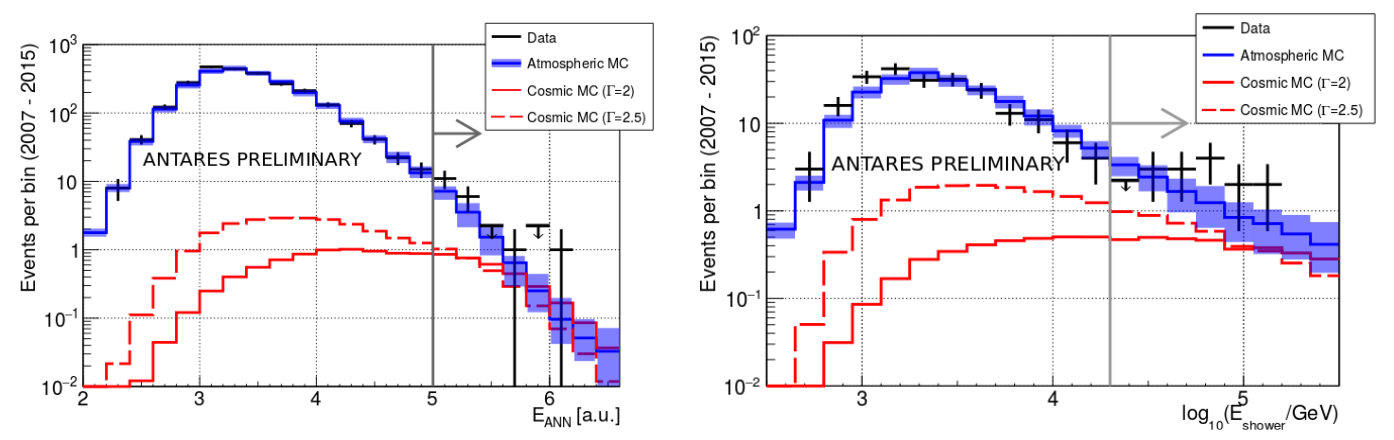

Figure 1: Distribution of the energy estimator for data (black crosses) and Monte Carlo after the event selection cut, before applying the energy-related selection shown as an arrow. Left panel for tracks, right for showers. The blue histograms show atmospheric events, with uncertainties reported by the shaded band (see text for details). Red distributions are for cosmic fluxes with spectral index $\Gamma=2.0$ (solid) or 2.5 (dashed). The normalisation of the cosmic signal is assumed equal to the best fit from IceCube measurements.

\subsection{Neutrinos inducing showers}

A dedicated reconstruction algorithm has been developed by the ANTARES Collaboration to reconstruct shower-like events [19] with high accuracy in direction and energy. A prefit is firstly performed; this provides the starting point for the final fitting as well as the value of the M-estimator evaluation, which is analogous to a $\chi^{2}$ for the hit distribution with respect to the shower hypothesis. This prefit is then followed by a maximum likelihood fit to obtain the shower parameters (direction and energy). The shower signature allows for the proper reconstruction of events only within a fiducial volume; differently from the track-channel, where the long muon track increases the effective volume and thus the event rate at the detector, a smaller number of cascade-like events are detectable in ANTARES. Nonetheless, in this channel, the atmospheric neutrino background is 
smaller by more than one order of magnitude, since it is mainly given by electron neutrinos, which are less abundant in atmospheric CR cascades. Finally, the energy resolution achievable for showerlike events allows for a better discrimination between the soft-spectra atmospheric neutrinos and the hard-spectra cosmic ones.

A preliminary event selection procedure, described in [28], is performed vetoing events that are already included in the track analysis, as well as events outside a fiducial volume and with poor reconstruction. After this event selection, the sample is still dominated by atmospheric muons. Three further signal-selecting procedures are defined based on: $a$ ) the total likelihood that the hit distribution induced by the event comes from an upward-going signal; $b$ ) the ratio between the likelihood of a track and a shower hypothesis to produce the reconstructed properties given the observed hit distribution; $c$ ) the ratio between the total measured charge of hits which are "ontime" with respect to the shower hypothesis, i.e. in space/time-correlation with the fitted vertex, and "early" hits arriving before the expectations from a shower hypothesis, thus compatible with a muon propagating through the instrumented volume [19].

The muon-rejecting procedure leaves about 0.008 atmospheric muon events per day in the whole sample. The atmospheric neutrino rate in this channel, after event selection, is approximately 0.1 events/day. The final selection is accomplished by rejecting events with reconstructed shower energy below $20 \mathrm{TeV}$, as a result of the MRF minimisation. The reconstructed shower energy distribution is reported in Figure 1. Overall, this event selection leads to an estimation of $10.5 \pm 4$ background events in the whole analysed livetime, when about 4 events are expected from a cosmic signal analogous to the IceCube observation.

\section{Results and conclusions}

The unblinding of the two samples yielded an observation of 33 (19 tracks and 14 showers) events overall. The expectation from Monte Carlo simulations of the background is 24 events (13.5 tracks and 10.5 showers).

A conservative estimation of the uncertainties on the background yields \pm 7 events. This uncertainty is computed taking into account the uncertainties related to the atmospheric neutrino flux normalisation, both for the conventional and prompt component, as well as on the contribution from different expectations on the behaviour of the primary CR knee [29]. In particular, the conventional flux is free to fluctuate by $\pm 25 \%$; the uncertainty on the prompt component is given by the highest and lowest prediction from [24]. It should be noted that the upper limit on the prompt component from IceCube measurement corresponds approximately to the Enberg et al. expectations [8]. The H3a model of the CR energy spectrum and composition is considered in the estimation of the background.

Analogously, the uncertainties on the normalisation of the background from wrongly reconstructed atmospheric muons are taken into account, allowing its normalisation to change by $30 \%$; in addition, as the final number of atmospheric muons in the data sample is extrapolated from Monte Carlo because of the limited statistics, also the uncertainties on this extrapolation are considered. This is completely negligible for the track channel, while it represents the largest contribution for the uncertainties in cascades. 


\begin{tabular}{cccc}
\hline Spectral Index & 90\%C.L. Upper Limit & 68\%Confidence Interval & Energy range \\
& $\Phi_{0}^{1 f}(1 \mathrm{GeV})$ & $\Phi_{0}^{1 f}(1 \mathrm{GeV})$ & \\
\hline \hline 2.0 & $3.4 \times 10^{-8}$ & $0.25-2.5 \times 10^{-8}$ & $40 \mathrm{TeV}-7 \mathrm{PeV}$ \\
2.5 & $1.9 \times 10^{-5}$ & $0.14-1.4 \times 10^{-5}$ & $30 \mathrm{TeV}-1.5 \mathrm{PeV}$ \\
\hline
\end{tabular}

Table 1: 90\% C.L. upper limit and 68\% confidence interval for the ANTARES diffuse flux analysis of tracks and showers combined, including systematics. The energy range in which the results are valid are computed from MC simulation accounting for the range in which $90 \%$ of the signal is contained for each signal spectral index.

Finally, different input for Monte Carlo simulations are considered in order to evaluate the effect of the detector response on the signal and background. This is achieved by changing the water properties as well as the efficiency of the optical modules. The corresponding effect is of the order of $10 \%$ at the energies considered here.

Once these effects are taken into account, the observation can be translated into a $68 \%$ confidence interval and a 90\% confidence level upper limit according to the method of [30] as reported in Figure 2 and Table 1. The energy ranges over which these results are valid is computed from Monte Carlo simulations, evaluating where the central $90 \%$ of signal events lies in the combined shower and track samples. The p-value of the observed excess is 0.15 . It should be noted that, though not significant, the observation is fully compatible with the expectation from an IceCubelike cosmic contribution in addition to the atmospheric backgrounds. A more detailed analysis of the spectral behaviour of the observed excess is foreseen.
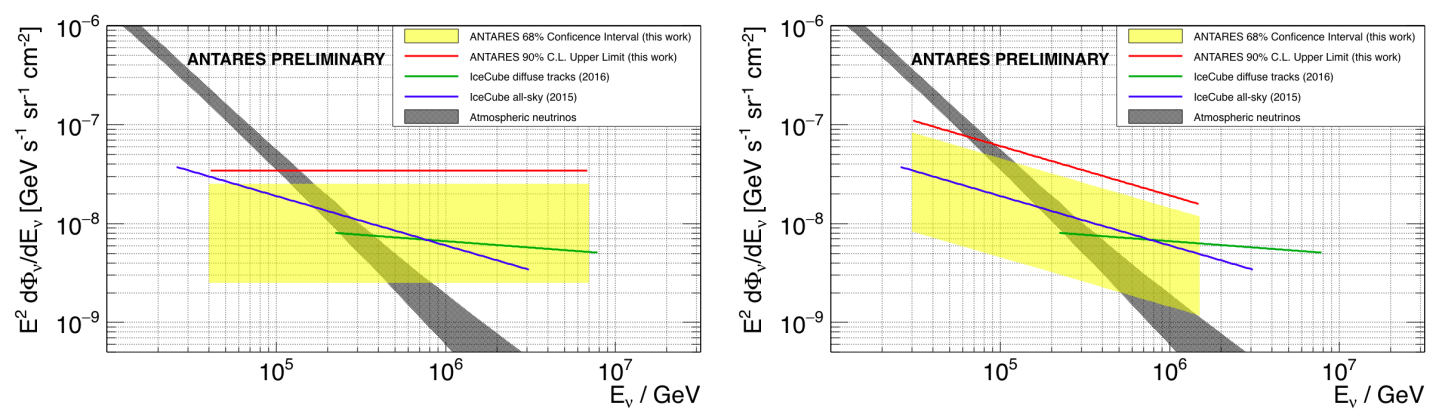

Figure 2: Upper limit at $90 \%$ C.L. (red line) and $68 \%$ condifidence interval (yellow band) for the combined track and shower analysis, including systematics. These are compared to the atmospheric neutrino background (gray shaded area - including uncertainties as in the text) and the measurement from IceCube for the Northern sky with tracks (green line) [8] and the combined all-sky analysis (blue line) [7]. Left panel is for a signal spectrum proportional to $\mathrm{E}^{-2}$, right for $\mathrm{E}^{-2.5}$.

\section{References}

[1] S.R. Kelner, F.A. Aharonian and V.V. Bugayov, Phys. Rev. D 74 (3): 034018 (2006).

[2] K. Mannheim, R. Protheroe and J.P. Rachen, Phys. Rev. D 63: 023003 (2001). 
[3] E. Waxman and J. Bahcall, Phys. Rev. D 59: 023002 (1998).

[4] E. Fermi, Phys. Rev. 75: 1169 (1949) and E. Fermi, Astrophys. J. 119: 1 (1954).

[5] A. Loeb and E. Waxman, JCAP 05: 003 (2006).

[6] M.G. Aartsen et al., Phys. Rev. Lett. 113: 101101 (2014).

[7] M.G. Aartsen, et al., Astrophys. J. 809: 98 (2015).

[8] M.G. Aartsen et al., Astrophys. J. 833 no.1: 3 (2016).

[9] A. Palladino, M. Spurio and F. Vissani, J. Cosmol. Astropart. Phys. 16(12): 045 (2016).

[10] M. Ageron et al., Nucl. Instrum. Meth. A 656: 11 (2011).

[11] S. Adrián-Martínez et al., Astrophys. J. 786: L5 (2014).

[12] S. Adrián-Martínez et al., Phys. Lett. B 760: 143 (2016).

[13] J.A. Aguilar et al., Phys. Lett. B 696: 16 (2011).

[14] S. Biagi et al., Proceedings of the 2013 Rencontres de Moriond (2014).

[15] J. Schnabel et al., POS(ICRC2015)1065 (2015).

[16] A. Albert et al., Eur. Phys. Journ. C 77: 419 (2017).

[17] A. Heijboer, arXiv:0908.0816 (2008).

[18] J. Schnabel et al., Nucl. Instr. and Meth. A 725: 106-109 (2013).

[19] T. Michael et al., POS(ICRC2015)1078 (2015).

[20] L.A. Fusco and A. Margiotta, Proceedings of the VLVnT 2015 Conference, Rome (2015).

[21] Y. Becherini et al., Astrop. Phys. 25: 1 (2006).

[22] G. Carminati et al., Comput. Phys. Commun. 179: 915-923 (2008).

[23] M. Honda et al., Phys. Rev. D 75: 043006 (2007).

[24] R. Enberg et al., Phys. Rev. D 78: 043005 (2008).

[25] S. Adrián-Martínez et al., Eur. Phys. Journ. C 73: 2606 (2013).

[26] G.C. Hill and K. Rawlins, Astrop. Phys. 19: 393 (2003).

[27] G.J. Feldman and R.D. Cousins, Phys. Rev. D 57: 3873-3889 (1998).

[28] A. Albert et al., submitted to Phys. Rev. D (2017), arXiv:1706.01857.

[29] T.K. Gaisser, T. Stanev, and S. Tilav, arXiv:1303.3565 (2013).

[30] J. Conrad et al., Phys. Rev. D 67: 012002 (2003). 